\title{
Supporting Information: Plasmon launching and scattering by silicon nanoparticles
}

\author{
Artyom Assadillayev, ${ }^{\dagger}$ Tatsuki Hinamoto, ${ }^{\ddagger}$ Minoru Fujii, ${ }^{\ddagger}$ Hiroshi Sugimoto, ${ }^{\ddagger}$ Mark \\ L. Brongersma, " and Søren Raza*,† \\ $\dagger$ Department of Physics, Technical University of Denmark, Fysikvej, DK-2800 Kongens \\ Lyngby, Denmark \\ $\ddagger$ Department of Electrical and Electronic Engineering, Kobe University, Rokkodai, Nada, \\ Kobe 657-8501, Japan \\ \Geballe Laboratory for Advanced Materials, 476 Lomita Mall, Stanford University, \\ Stanford, CA 94305, USA \\ E-mail: sraz@dtu.dk
}

Figure S1: Distinguishing the spectrally-close EQ and MH Mie resonances.

Figure S2: Additional spatial EELS maps for Figure 2 in the manuscript.

Figure S3: Mie resonances in silicon nanoparticles on a $10 \mathrm{~nm}$ thin gold film.

Figure S4: Plane-wave excitation of the hybrid dielectric-plasmon mode.

Figure S5: SPP coupling efficiency of the hybrid dielectric-plasmon mode as a function of gold thickness.

Figure S6: Plasmon reflection for different interference orders.

Figure S7: Simulated plasmon reflection from silicon nanowires.

Figure S8: Surface plasmon reflection: EELS signal outside the silicon nanoparticle. 


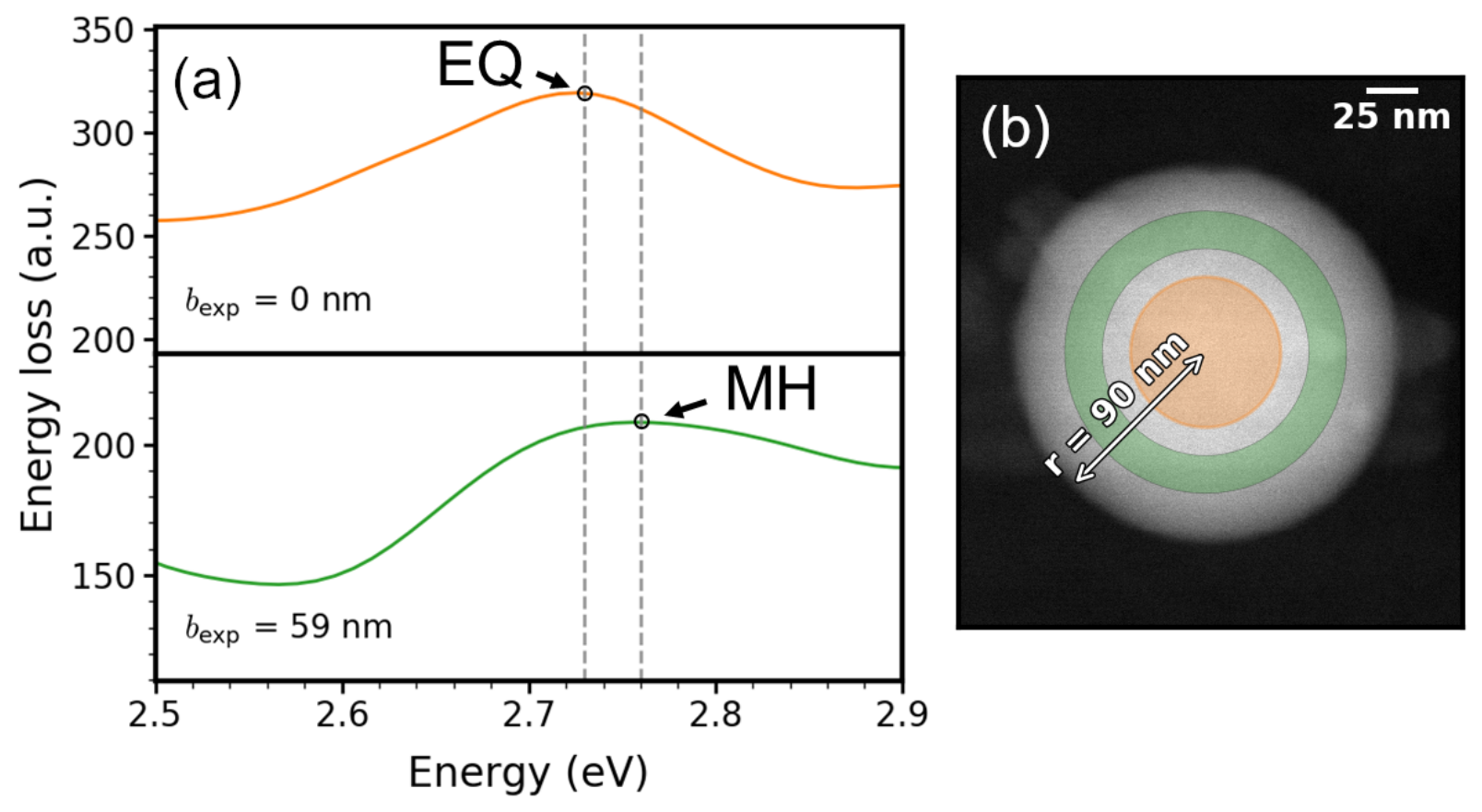

Figure S1: Distinguishing the spectrally-close EQ and MH Mie resonances. (a) Experimental EELS spectra of a silicon nanoparticle $(r=90.5 \pm 2.0 \mathrm{~nm})$ on a thin silicon nitride membrane at the electron beam positions indicated in (b). Careful positioning of the electron beam enables us to shift the EELS peak to either the EQ or MH resonance energies. This allows us to measure both resonance energies from a single silicon nanoparticle, even though their energies are closer than the energy resolution of our EELS setup. 

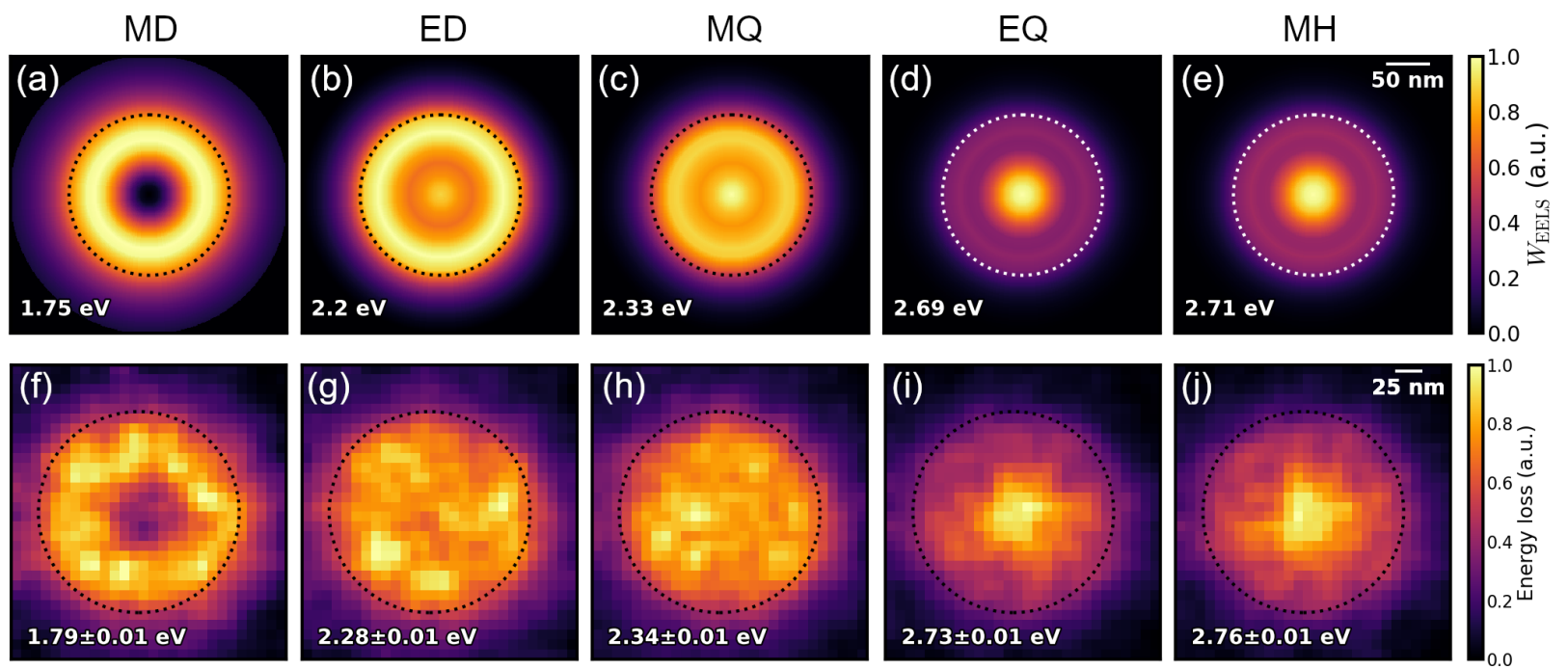

Figure S2: Additional spatial EELS maps for Figure 2 in the manuscript. (a-e) Simulated EELS maps for the EELS spectra convoluted with a Lorentzian function. A maximum appears in the nanoparticle center for the ED and MQ modes. This leads to closer correspondence with the experimental EELS maps shown in Figure 2. (f-j) Experimental EELS maps before spatial integration along the azimuth angle. While similar features may be observed in the unprocessed EELS maps, it is clear that exploiting the spherical symmetry of the nanoparticles to perform an azimuthal integration results in EELS maps with markedly improved signal-to-noise ratio. 


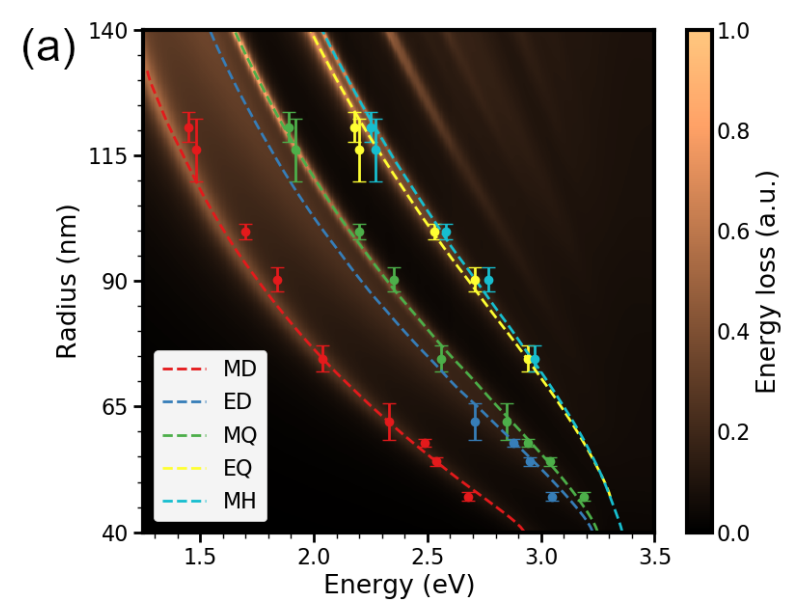

(b)
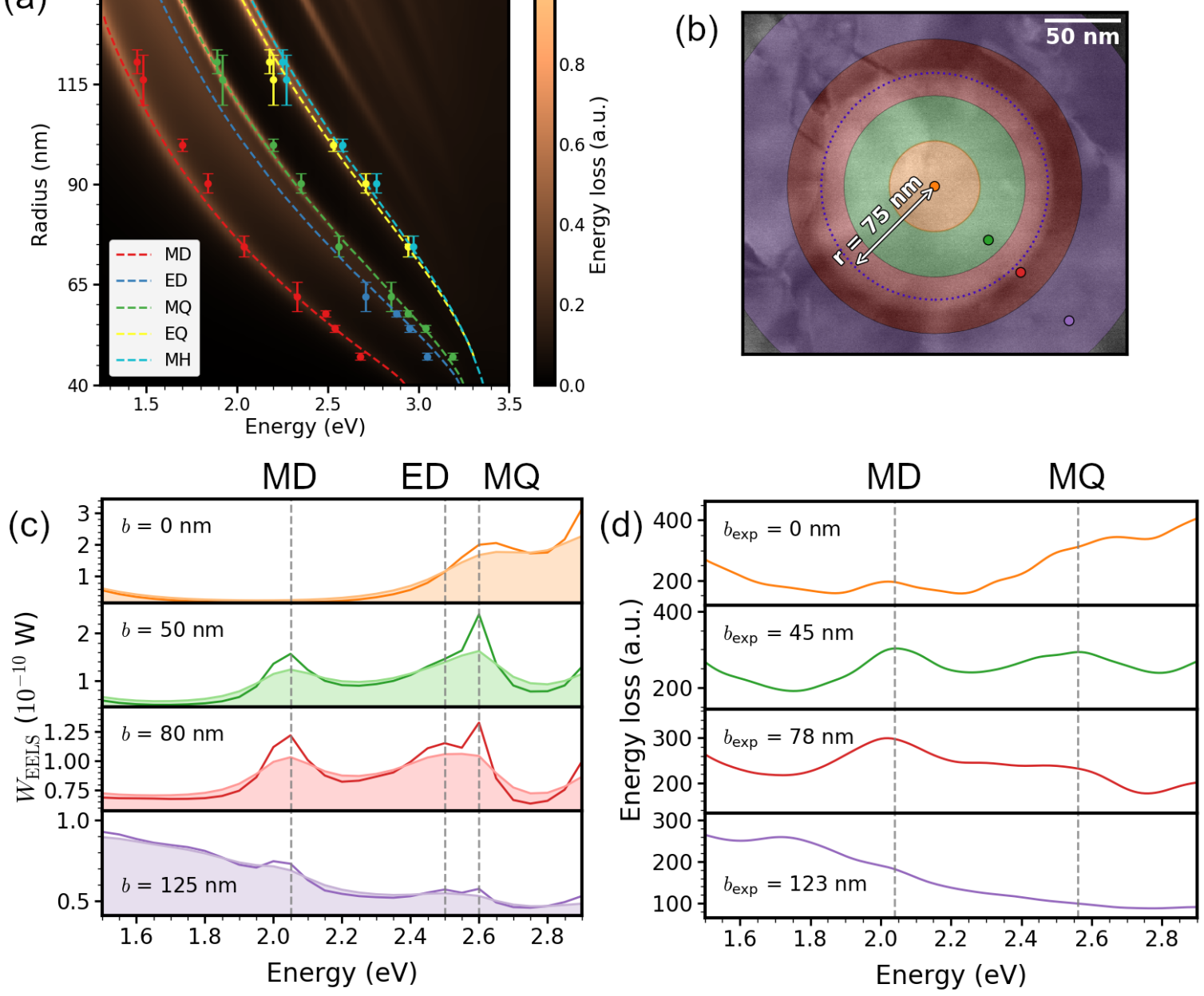

Figure S3: Mie resonances in silicon nanoparticles on a $10 \mathrm{~nm}$ thin gold film. (a) Measured resonance energies as a function of particle radius for the first five Mie resonances in silicon nanoparticles on the gold-silicon nitride stack. The colour plot shows the EELS simulation of a vacuum-embedded silicon sphere for an impact parameter $b=r+20 \mathrm{~nm}$ and the dashed curves indicate the free-space theoretical Mie resonance energies. The strong agreement between theory and experiments show that the Mie resonances remain largely unaffected by the presence of the thin gold film. (b) STEM image of the nanoparticle, where the colored areas represent the integration regions for the experimental EELS signal, and the colored points are the position of the electron beam in the simulation. (c) Simulated EELS spectra of a silicon nanoparticle $(r=75 \mathrm{~nm})$ on a thin gold-silicon nitride stack at different impact parameters $b$. The area curves depict the simulated spectra convoluted with a Lorentzian function. (d) Experimental EELS spectra for a particle with radius $r=74.5 \pm 2.6 \mathrm{~nm}$ acquired from the regions shown in (b). The EELS spectra show that the Mie modes can be safely identified when the electron beam is positioned inside the particle. As the beam is moved outside the nanoparticle, the EELS signal from the SPP of the gold film starts to dominate the spectrum, which masks the signal from the ED mode in larger particle sizes. For smaller particle sizes, the ED resonance energy occurs at much larger energies than the $\mathrm{SPP}$, allowing us to identify the ED resonance. 

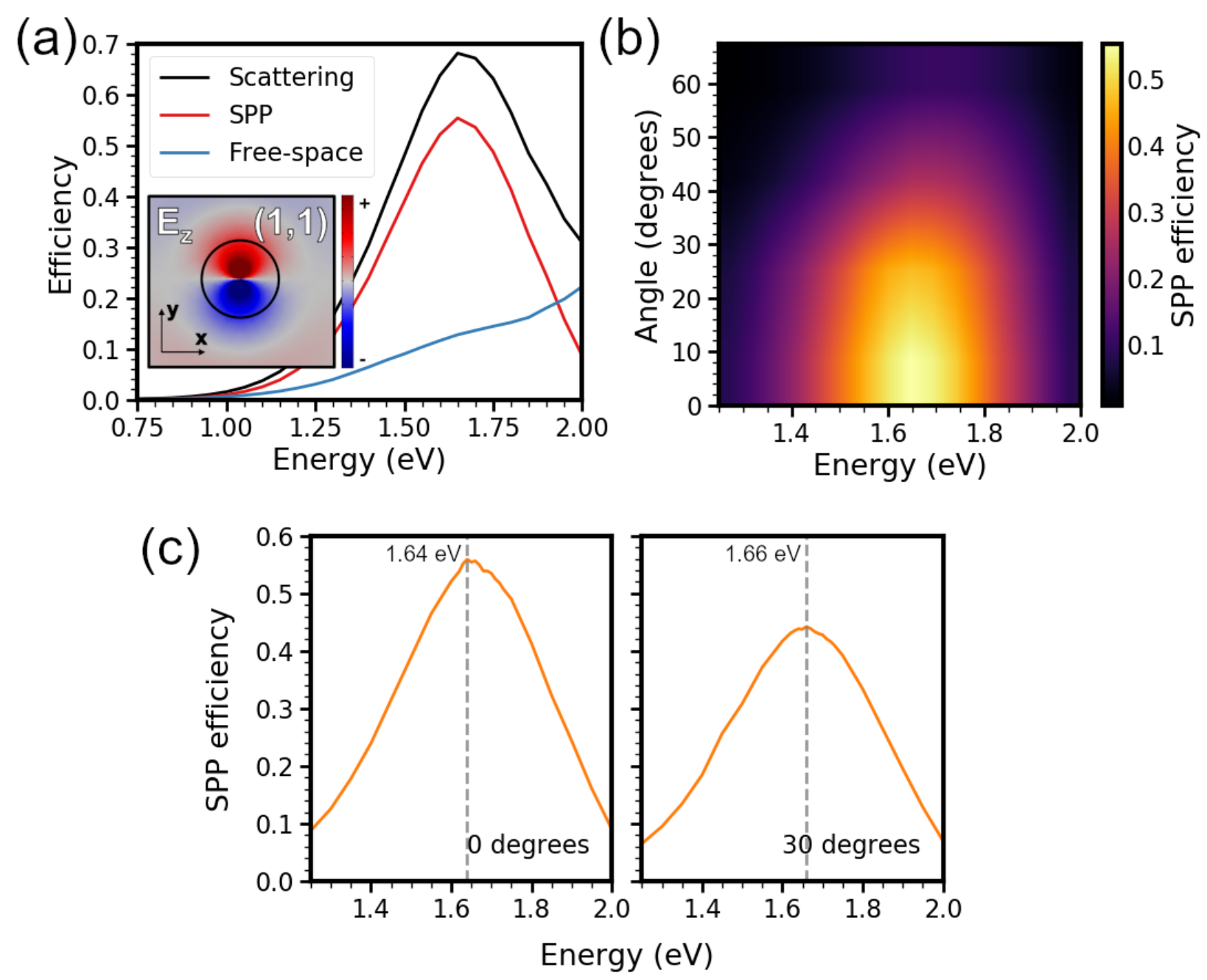

Figure S4: Plane-wave excitation of the hybrid dielectric-plasmon mode. (a) Plane-wave scattering efficiency of a silicon nanoparticle with radius $r=55 \mathrm{~nm}$ on top of a gold-silicon nitride stack. The scattering efficiency is decomposed into SPP and free-space radiation contributions. The incident plane wave impinges normal to the gold-silicon nitride stack. The inset shows the $z$ component of the electric field of the $(1,1)$ hybrid dielectric-plasmon mode in the $x y$-plane at the top gold surface. (b) SPP coupling efficiency as a function of the angle of incidence relative to the perpendicular plane of the gold-silicon nitride stack. (c) SPP efficiency for normally-incident and $30^{\circ}$-incident impinging plane-wave excitation. The SPP efficiency peak shows a delicate shift, consistent with the EELS measurements in Figure 4, accompanied by a modest decrease in efficiency due to a slight re-orientation of the induced electric dipole moment. 

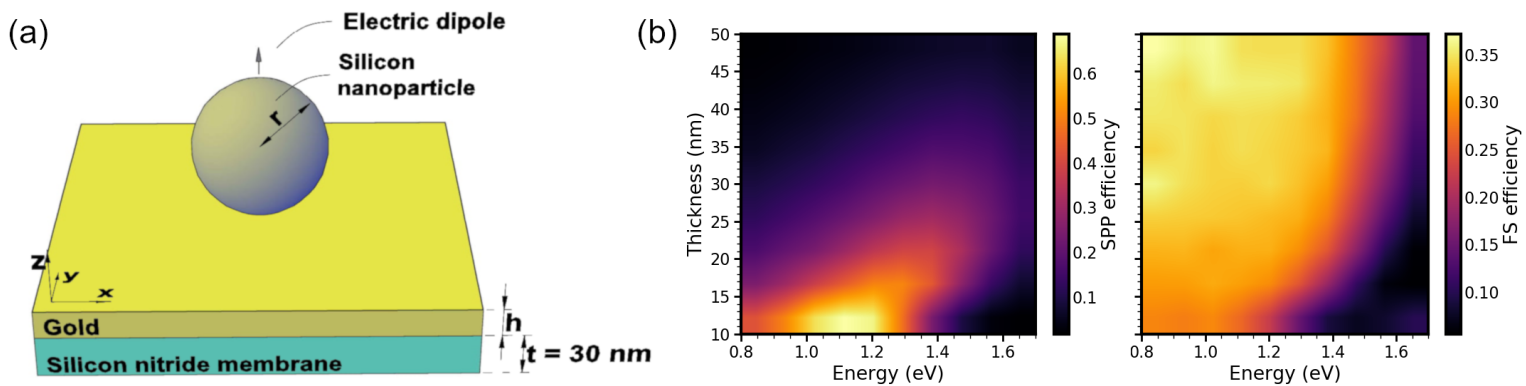

Figure S5: SPP coupling efficiency of the hybrid dielectric-plasmon mode as a function of gold thickness. (a) Schematic of a silicon nanoparticle placed on the gold-silicon nitride stack, which is probed by an electric dipole oriented along the $z$ direction and located above the centre of the nanoparticle at a $1 \mathrm{~nm}$ gap. (b) Efficiency of SPP and free-space (FS) coupling of the $(0,1)$ hybrid dielectric-plasmon mode for increasing gold layer thickness. The efficiency is taken as a ratio of the SPP or FS power to the power radiated by the electric dipole in vacuum. The results show the increasing (decreasing) SPP (FS) efficiency for decreasing film thickness, highlighting the importance of thin gold films for efficient SPP coupling.

(a) 1 order
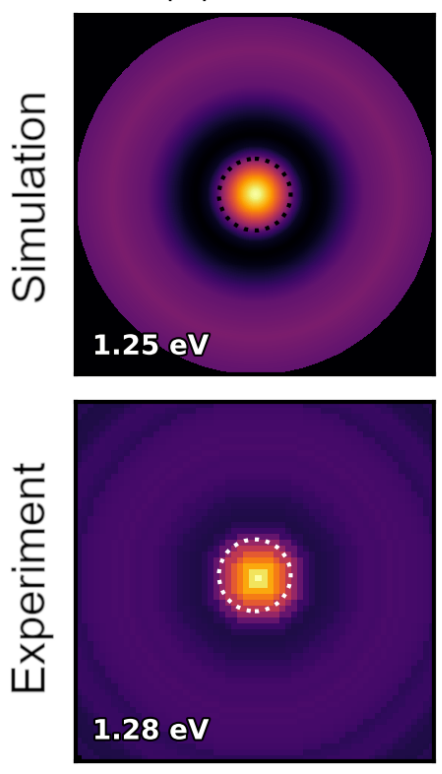

(b) 2 order

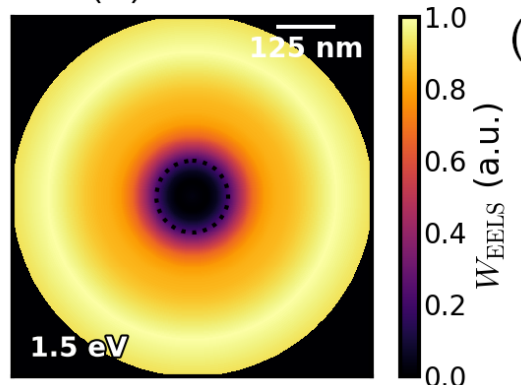

(c)
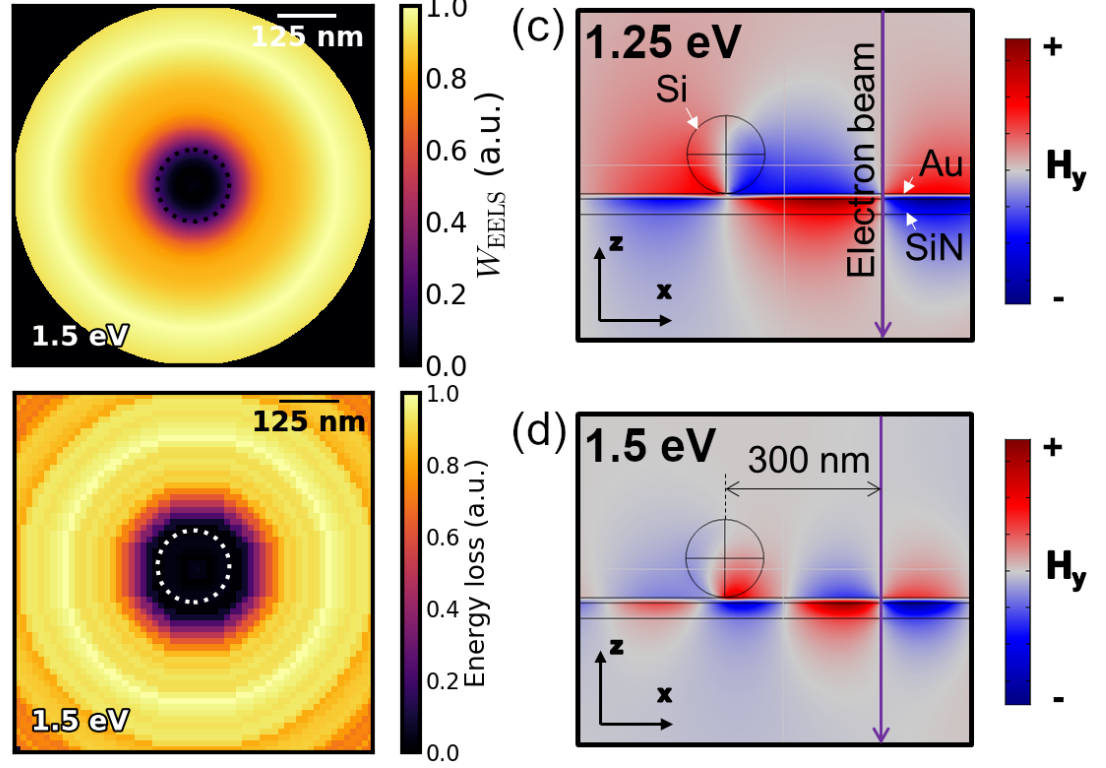

(d)

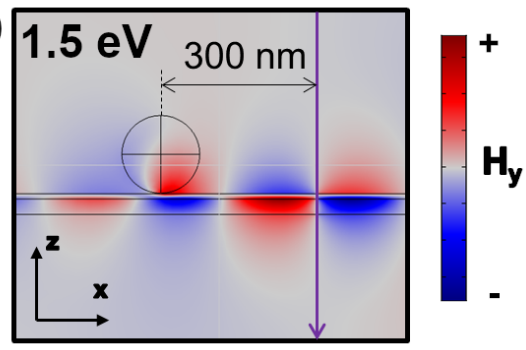

Figure S6: Plasmon reflection for different interference orders. (a,b) Simulated (top) and experimental (bottom) EELS maps of the plasmon reflection at two separate energies, which correspond to either (a) the first-order reflection (half-wavelength of SPP) or (b) secondorder reflection (one-wavelength of SPP). The nanoparticle radius is $r=75 \mathrm{~nm}$. (c,d) Crosssectional magnetic field profiles of the reflected SPP mode for first and second interference orders, respectively. In both cases, the reflection occurs at the nanoparticle centre. 


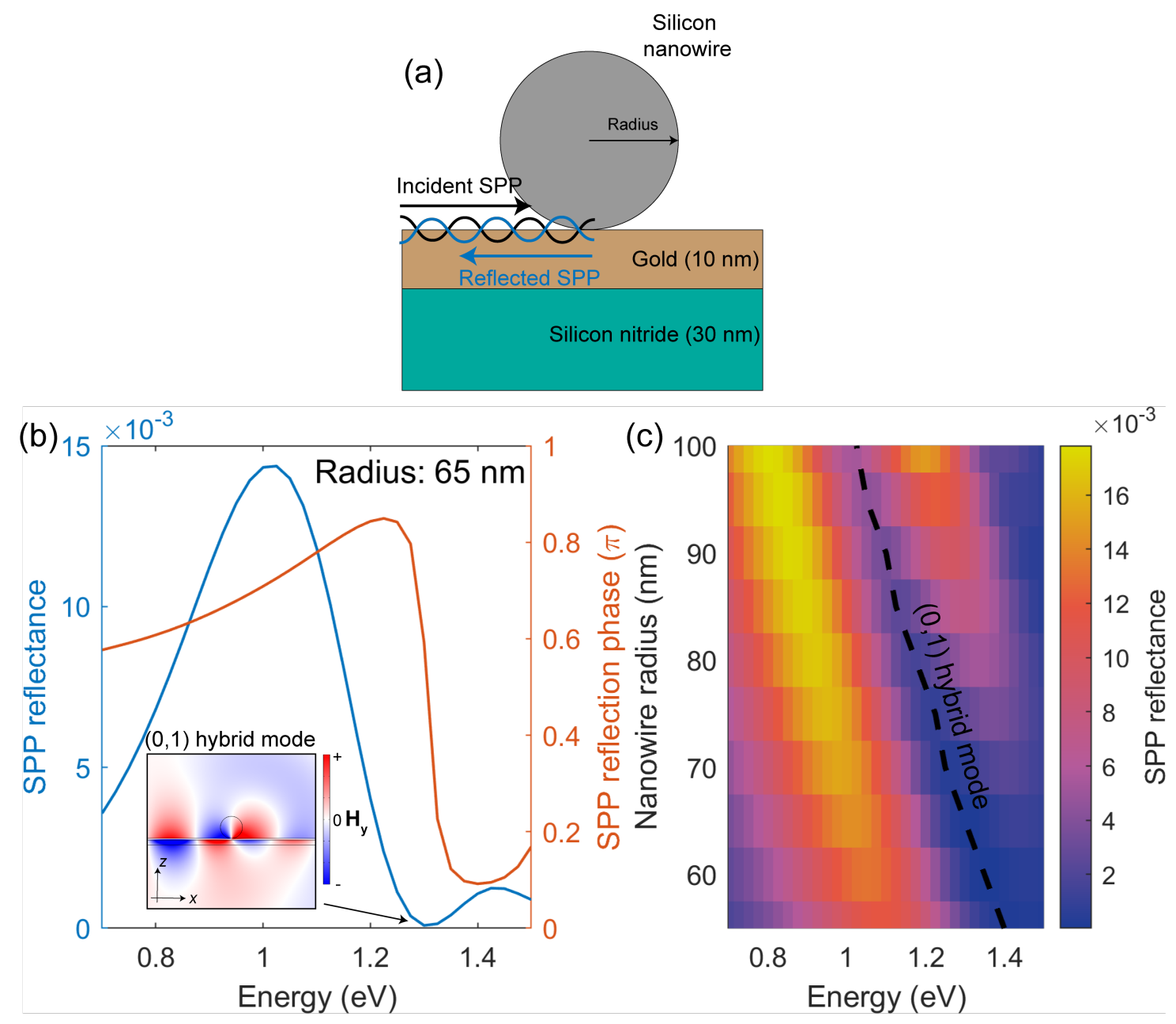

Figure S7: Simulated plasmon reflection from silicon nanowires. (a) The SPP supported by the gold-silicon nitride stack is incident on a silicon nanowire (2D). The incident SPP scatterers into different radiations channels, including reflected and transmitted SPPs as well as free-space radiation. Here, we track the reflected SPP as this is relevant for the EELS measurements in Figure 4. (b) SPP reflectance and reflection phase for a $65 \mathrm{~nm}$ radius silicon nanowire. The reflection is evaluated at the at the nanowire centre. The reflectance dip is accompanied by a rapid phase variation, signifying the excitation of the $(0,1)$ hybrid dielectric-plasmon mode. The inset shows the out-of-plane magnetic field profile at the reflectance dip, which matches well with the field profile shown in Figure 3. The hybrid mode enables efficient outcoupling of the SPP, hence the low reflectance. (c) SPP reflectance map for varying nanowire radius, which shows that the hybrid mode redshifts for increasing radii, in accordance with the EELS measurements in Figure 4. 

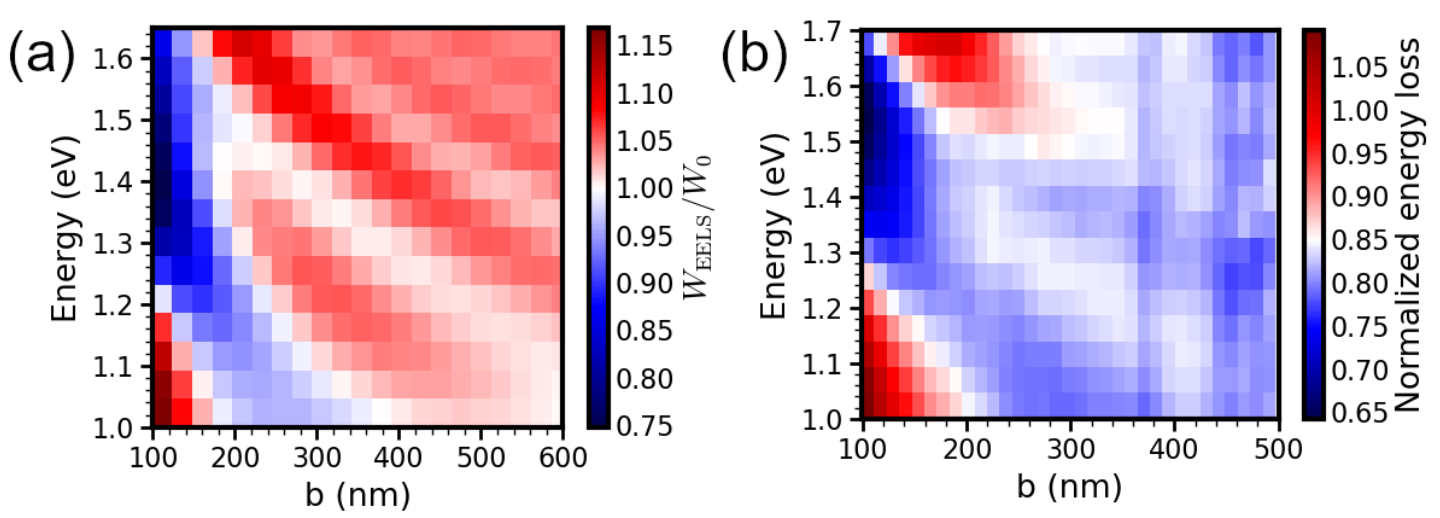

Figure S8: Surface plasmon reflection: EELS signal outside the silicon nanoparticle. (a) Simulated and (b) experimental normalized EELS signal as a function of energy and electron beam position $b$. The silicon nanoparticle has a radius of $r=75 \mathrm{~nm}$ and is located at the origin. In both simulations and measurements, the EELS signal outside the silicon nanoparticle is normalized to the signal from the gold-silicon nitride stack (i.e., without the particle). The regions of intense EELS signal correspond to maxima in the interference pattern due to the SPP reflection by the nanoparticle. The intense EELS signal seen at low energies and small impact parameters (lower left corners) is due to the hybrid mode. A weak drop in the intensity of the interference pattern is observed at approximately $1.4 \mathrm{eV}$ $(1.45 \mathrm{eV})$ in the simulations (measurements), which could potentially be due to outcoupling of the SPP to free space radiation. 